\title{
The Outer Galactic Halo As Probed By RR Lyr Stars \\ From the Palomar Transient Facility + Keck
}

\author{
Judith Cohen $^{1}$, Branimir Sesar ${ }^{1,2}$, Sophianna Banholzer ${ }^{1}$, the PTF \\ Collaboration $^{1}$ \\ ${ }^{1}$ California Institute of Technology, Palomar Observatory, \\ Mail Code 249-17, Pasadena, Ca., 91125, USA \\ email: jlc@astro.caltech.edu \\ ${ }^{2}$ Max Planck Institute for Astronomy, \\ Konigstühl 17, D-69117, \\ Heidelberg, Germany \\ email: bsesar@mpia.de
}

\begin{abstract}
We present initial results from our study of the outer halo of the Milky Way using a large sample of RR $\operatorname{Lyr}(a b)$ variables datamined from the archives of the Palomar Transient Facility. Of the $464 \mathrm{RR}$ Lyr in our sample with distances exceeding $50 \mathrm{kpc}, 62$ have been observed spectroscopically at the Keck Observatory. $v_{r}$ and $\sigma\left(v_{r}\right)$ are given as a function of distance between 50 and $110 \mathrm{kpc}$, and a very preliminary rather low total mass for the Milky Way out to $110 \mathrm{kpc}$ of $\sim 7 \pm 1.5 \times 10^{11} M_{\odot}$ is derived from our data.
\end{abstract}

Keywords. The Galaxy, Galaxy: halo, stars: variables: RR Lyrae

\section{Introduction}

We present initial results from our study of the outer halo of the Milky Way (MW) using a large sample of RR $\operatorname{Lyr}(a b)$ variables datamined for the archives of the Palomar Transient Facility (PTF) (Law et al. 2009, Rau et al. 2009) (P.I. S. R. Kulkarni of Caltech). RR Lyr are old low-mass pulsating stars with distinctive light curves, amplitudes at $V$ of $\sim 1 \mathrm{mag}$, and periods of $\sim 0.5$ days. These characteristics makes them fairly easy to distinguish in a wide field, multi-epoch optical imaging survey of moderate duration if the survey cadence is suitable. Their most desirable characteristic is that they can be used as standard candles. Accurate luminosities, which have only a small metallicity dependence, can be inferred directly from the light curves, and these stars, with $M_{V} \sim+0.6 \mathrm{mag}$, are fairly luminous and hence can be detected at large distances.

Using machine learning techniques we have isolated a sample of $464 \mathrm{RR}$ Lyr from the PTF. These were found by searching for PTF fields which had enough epochs of observation (25 minimum) in the $\mathrm{R}$ filter. A total of roughly $10,000 \mathrm{deg}^{2}$ on the sky met this requirement and was searched. The regions of known outer halo objects (i.e. dwarf galaxies, globular clusters, and known halo streams) were excluded. The search criterion was variability, and the sample was refined by requiring the derived period and amplitude to have values appropriate for RR Lyr. Since no observing time was assigned for this purpose, we are effectively datamining the PTF archive, and our sample lies in random pencil beams through the halo, each a PTF tile (area on sky: $7.6 \mathrm{deg}^{2}$ ).

Our sample begins at a brightness corresponding to a distance of $50 \mathrm{kpc}$, and extends out to $\sim 110 \mathrm{kpc}$, after which RR Lyr are too faint to pick out with PTF data. The selection procedure was carried out in 2012 ; at present the PTF $+\mathrm{iPTF}$ variable star database 


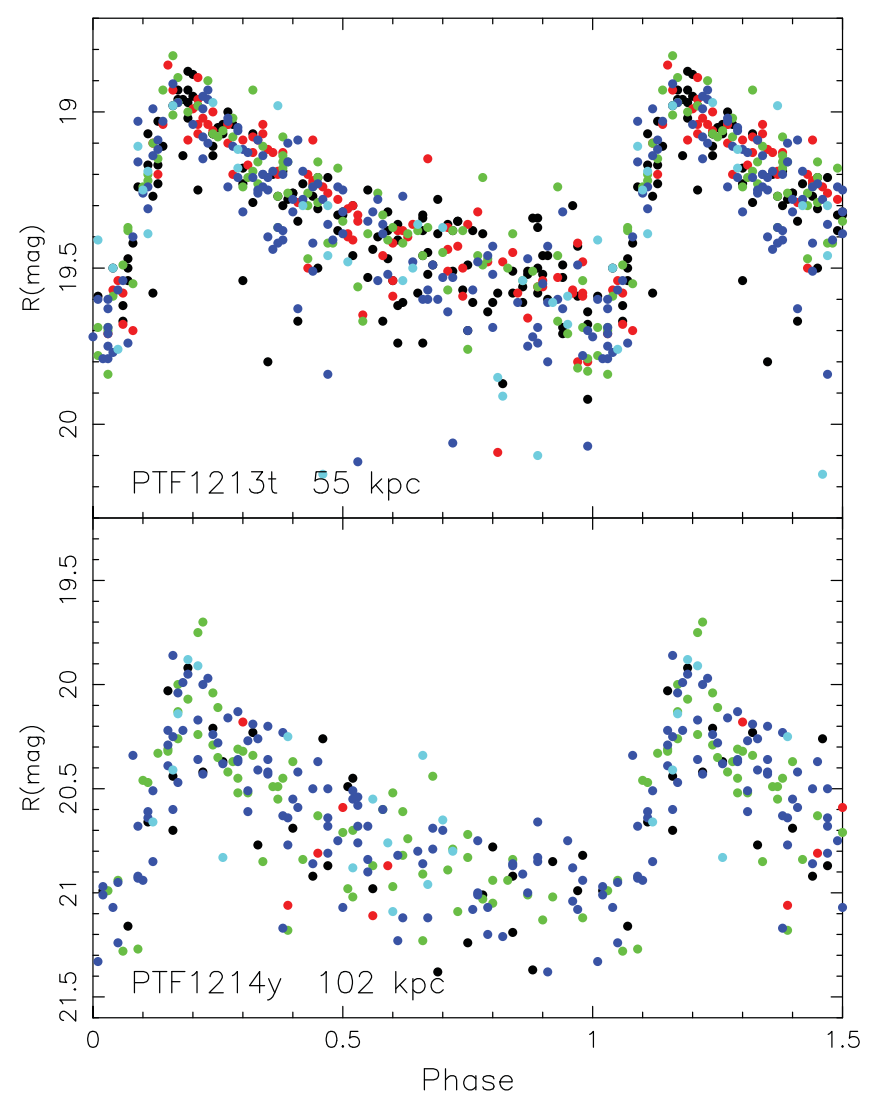

Figure 1. Examples of phased PTF light curves: Top: A RR Lyr at a distance of $55 \mathrm{kpc}$ with 468 measurements with the R filter in the PTF database. Bottom: A RR Lyr at a distance of $102 \mathrm{kpc}$ with $219 \mathrm{R}$ measurements in the PTF database. Note that prior to the addition of 2 years of data to the PTF variable star database in early 2015 , this star had only 61 detections at $\mathrm{R}$ in the PTF database. The color scheme denotes the year of observation, with the first day set to the first night the object was observed by the PTF. Black $=$ year 1 , red $=$ year 2 , green $=$ year 3 , blue $=$ year 4 , etc using the PGPLOT color routine. The sample selection was carried out using only the first two years of PTF data.

contains photometry through Dec 2014, i.e. two more years of data, none of which was used to select the current sample. A more detailed description of the search method is given in Sesar et al. (2014). Given this is a variability search, the only contaminants with colors similar to those of RR Lyr are quasars (QSOs).

Fig. 1 (top) shows the light curve of one of the brighter RR Lyr in our sample $(r=$ $55 \mathrm{kpc})$ as well as that of one of the most distant RR Lyr found to date $(r=102 \mathrm{kpc})$ (bottom).

\section{Radial Velocity Measurements}

A spectroscopic campaign to obtain radial velocities for RR Lyr candidates began at the Keck Observatory with the Deimos spectrograph (Faber et al. 2003) in the spring of 2014. RR Lyr are pulsating variable stars, hence their observed radial velocities need to be corrected for the motion of the atmosphere. To measure the center-of-mass velocity $v_{r}$ of RR Lyr stars, we use the Balmer $\mathrm{H} \alpha$ line and the method described by Sesar (2012). 


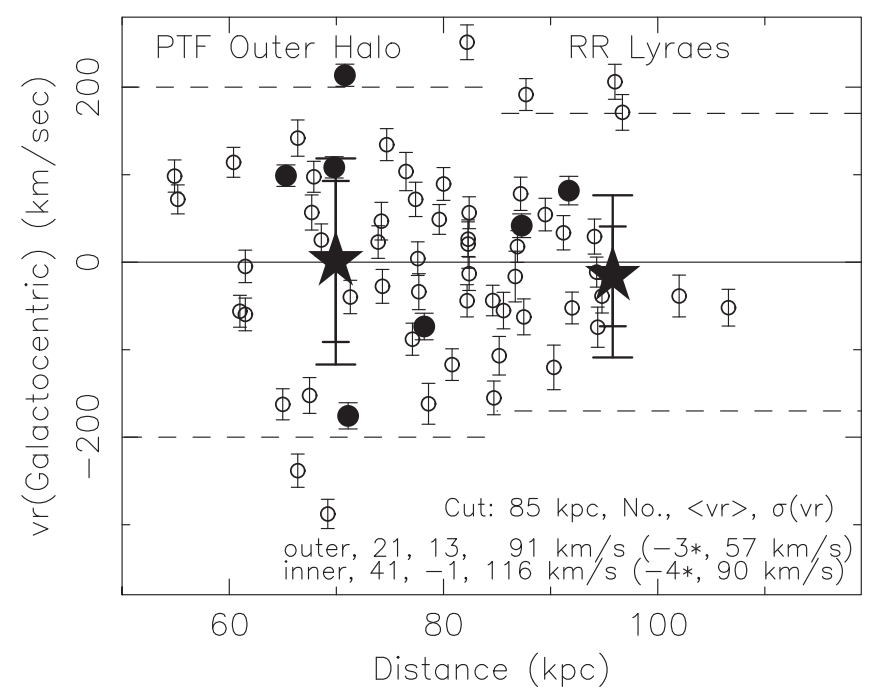

Figure 2. Radial velocities as a function of heliocentric distances are shown for our sample of 62 RR Lyr selected from the PTF with Keck/Deimos moderate resolution spectra. The borders of the regions (both high and low $v_{r}$ ) considered outliers are indicated by the dashed horizontal lines. The text at the bottom right gives the number of RR Lyr, mean and $\sigma$ (with $20 \mathrm{~km} \mathrm{~s}^{-1}$ removed in quadrature for observational uncertainties) for the inner and for the outer sample, cut at $85 \mathrm{kpc}$, as well as these with the largest outliers removed ( 3 stars for the outer region, and 4 for the closer sample). Filled circles denote stars with two Deimos spectra, open circles have only one spectrum. One $\sigma$ error bars are shown for each RR Lyr. The two large stars denote the means for the inner and outer sample with the outliers removed; their two error bars correspond to $\sigma$ for the full sample, and that for the cropped sample.

Typical uncertainties in center-of-mass velocities, including both observational and phase correction, are 15 to $20 \mathrm{~km} \mathrm{~s}^{-1}$ for a single Deimos spectrum.

Fig. 2 shows $v_{r}$ vs $r$ for the 62 RR Lyr with Keck/Deimos spectra. We cut the sample at $85 \mathrm{kpc}$, and assume an uncertainty in each individual measurement of $20 \mathrm{~km} \mathrm{~s}^{-1}$. We find a mean $v_{r}$ for the inner $41 \mathrm{RR}$ Lyr of $-1 \mathrm{~km} \mathrm{~s}^{-1}$ with $\sigma=116 \mathrm{~km} \mathrm{~s}^{-1}$, while for the outer 21 stars, the mean is $+13 \mathrm{~km} \mathrm{~s}^{-1}$ with $\sigma\left(v_{r}\right)=91 \mathrm{~km} \mathrm{~s}^{-1}$. The mean of $v_{r}$ falls close to 0 , suggesting that our corrections to observed radial velocities are appropriate.

When 4 major outliers ( 2 high and 2 low) are deleted from the inner sample, $\sigma$ falls to $90 \mathrm{~km} \mathrm{~s}^{-1}$ for the inner sample. Deleting three high outliers from the outer sample leaves $18 \mathrm{R}$ Lyr beyond $85 \mathrm{kpc}$, and $\sigma$ falls to $57 \mathrm{~km} \mathrm{~s}^{-1}$, a remarkably low dispersion. We are not yet certain whether the outliers arise from substructures within the outer halo.

The velocity dispersion as a function of radius for the Milky Way halo is shown in Fig. 3, where we compare our work to selected values from the recent literature. The small stars denote our entire sample, cut at $85 \mathrm{kpc}$, and the larger stars show our sample of outer halo RR Lyr cleaned of outliers; recall that the outliers comprise only about $10 \%$ of our total sample. The star symbols are plotted at the median distance of the inner and of the outer sample. The velocity dispersions found for the inner halo from recent studies are also shown: from SDSS blue horizontal branch (BHB) stars (Xue et al. 2004), from SDSS K giants (Xue et al. 2014) as well as a sample of halo high velocity stars from Brown et al. (2010). Most of these other samples are confined to distances less than $80 \mathrm{kpc}$. The only attempt to reach the distances probed by the outer part of our sample is that of Deason et al. (2012). Our results agree quite well with the value they published of $\sigma\left(v_{r}\right)$ of 50 to $60 \mathrm{~km} \mathrm{~s}^{-1}$ at distances of $\sim 110 \mathrm{kpc}$, rising to $\sim 90 \mathrm{~km} \mathrm{~s}^{-1}$ at distances 


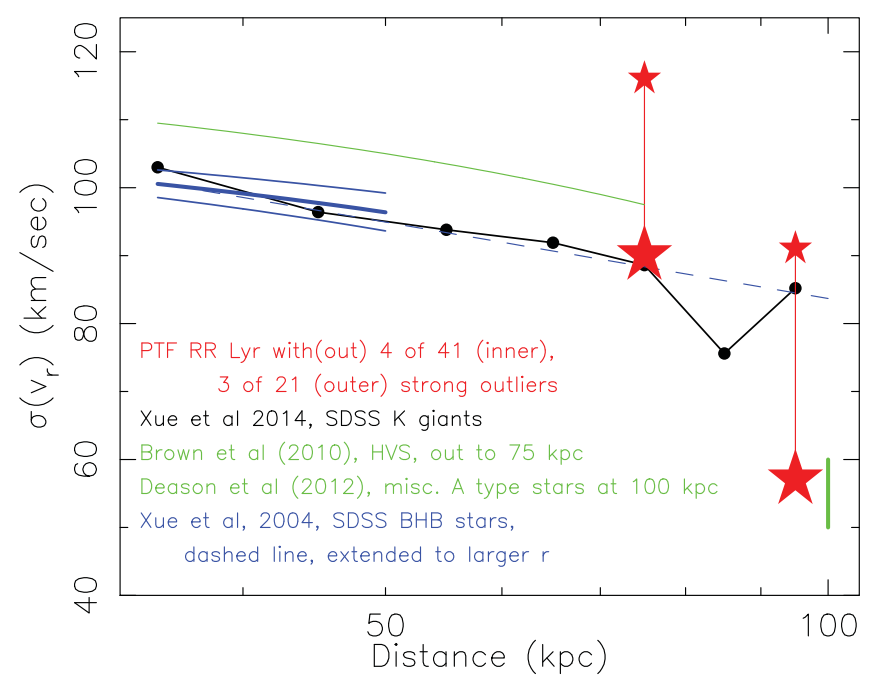

Figure 3. $\sigma\left(v_{r}\right)$ is shown as a function of $\log (r)$ for our inner and outer sample of RR Lyr (split at $85 \mathrm{kpc}$ ), with and without eliminating the single strong outliers. Values from Xue et al. 2008), whose extrapolation to larger $r$ is indicated by a dashed line, Xue et al. (2011, 2014), Brown, Geller \& Kenyon (2014), and Deason et al. 2012b), are shown. The text within the figure indicates the colors used for each reference and the nature of the sample in each case.

of $70 \mathrm{kpc}$. We agree reasonably well with the result of Brown et al. (2010) for $\sigma\left(v_{r}\right)$ as a function of $r$ from the MMT high velocity star survey, extending out to $75 \mathrm{kpc}$, when their non-parametric method to eliminate outliers is used (see their Fig. 6).

\section{Implications}

One of our goals is to determine the radial distribution of the outer halo of the Milky Way using RR Lyrae as tracers of its stellar population, and to use this information to derive the total mass of the MW. We calculate distances assuming a fixed $M_{R}$ of $+0.6 \mathrm{mag}$ as the flux-averaged mean over the period, then correct for interstellar reddening assuming that the variable is so distant that the full reddening from the maps of Schlegel, Finkbeiner \& Davis (1998) applies.

Fig. 4 shows a histogram of the full sample of 464 candidate RR Lyr beyond $50 \mathrm{kpc}$ as a function of $\log (r)$, where $r$ is the heliocentric distance. Both axes have logarithmic scales. The vertical axis is the number of variables in bins equally spaced in distance starting at $50 \mathrm{kpc}$, with $2.8 \mathrm{kpc} /$ bin. These counts, accumulated over a solid angle on the sky of $\Omega \sim 10,000 \mathrm{deg}^{2}$, represent $\rho(r)(\Omega / 4 \pi) 4 \pi r^{2} \Delta r$. We fit a power law $\rho \propto r^{-\gamma}$, over the range of $r$ we cover, i.e. out to just over $100 \mathrm{kpc}$. Therefore a linear fit of the $\log$ (counts) vs $\log \left(R_{G C}\right)$ will yield a slope that is $-\gamma+2$. Our best fit slope is $\gamma=3.8 \pm 0.3$.

The completeness corrections are very difficult to calculate because the number of epochs observed varies greatly among the PTF fields, depending on which (other) project requested observations of the field; the Galactic halo project has until recently been allocated no P48 imaging time at all; we have simply used observations acquired for other PTF subprojects and thus have no control over the cadence of observing nor the fields observed. Furthermore, PTF observations at the P48 are carried out as long as the dome can safely remain open irrespective of clouds. Thus the limiting magnitude of each exposure of a given field has a very wide range, with clouds (i.e. a bright limiting magnitude) more common than exceptional nights which are perfectly clear and with 


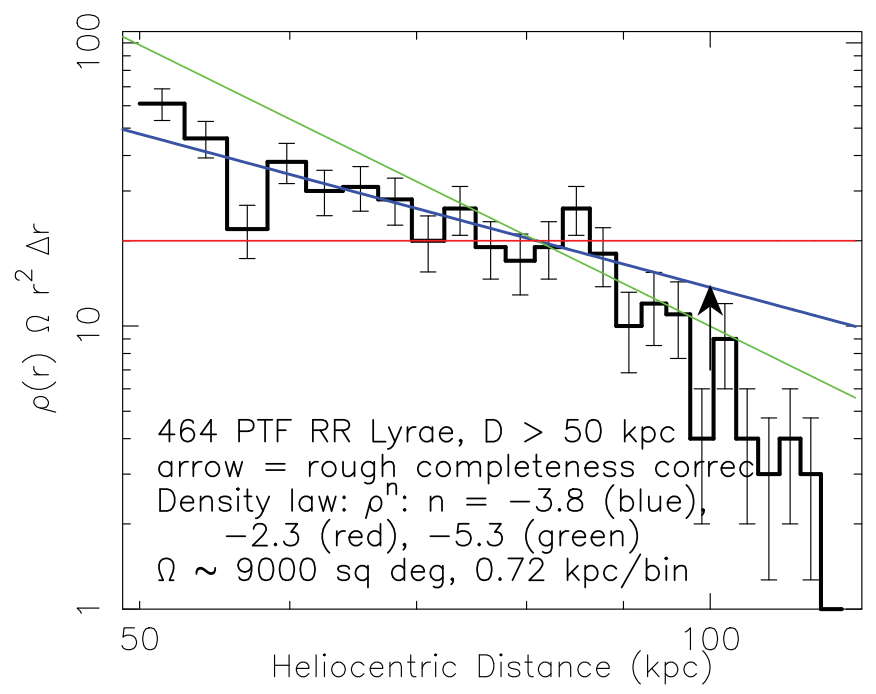

Figure 4. The number density of RR Lyr as a function of distance is shown together with several power law fits which suggest $\gamma \approx 3.8$. A lower limit to the incompleteness correction at a distance of $100 \mathrm{kpc}$ is shown as a vertical arrow.

good seeing, which have the faintest limiting magnitudes. An RR Lyr at $100 \mathrm{kpc}$ has a mean $\mathrm{R}$ of 20.6, close to the limit of the PTF survey, so that in many PTF images it will not be detected. To aquire a specified minimum number $N$ of $\mathrm{R}$ detections, where $N$ is set by our search procedure to identify a candidate RR Lyr, will thus require many more epochs of observation with PTF of its field than would be the case for a RR Lyr at $50 \mathrm{kpc}$ in the same field. We indicate our best guess of a lower limit to the completeness correction arising from this issue at $100 \mathrm{kpc}$ by the vertical arrow in Fig. 4. Other such issues will further increase the completeness correction above $90 \mathrm{kpc}$.

The only effort that reaches out to the radial range covered by our RR Lyr sample is that of Deason et al. (2014), who claim there is a very steep outer halo profile, with $\gamma \sim 6$ beyond $r=50 \mathrm{kpc}$ and even steeper slopes $\gamma \sim 6-10$ at larger radii. Their sample of $\sim 5200$ stars is contaminated by QSOs; a photometric separation is not sufficient and cuts out many BHB stars.

We cannot reproduce the extremely steep decline in $n(r)$ that they claim to observe. We believe that with our RR Lyr sample selected through variability, the precise distances we obtain for our RR Lyr stars, and our low QSO contamination, that our result that $\rho \propto r^{-3.8 \pm 0.3}$ for $50<r<100 \mathrm{kpc}$ is correct. Most other recent analyses, i.e. Xue et al. (2015), Brown et al. 2010, find $\gamma$ between 3.5 and 4.5 from $r=20 \mathrm{kpc}$ out to the limit of their data, between 60 and $80 \mathrm{kpc}$; see e.g. Fig. 1 of Gnedin et al. (2010).

As a rough indication of the total mass of the MW, we assume a spherical halo, ignoring the subtleties in the inner part of the MW of the thick and thin disk. We also ignore the difference between our heliocentric distances and galactocentric distances. The standard way to obtain the total enclosed mass given a set of tracers, be they globular clusters or RR Lyr or any other low mass objects in the outer halo of a massive galaxy, is to solve the Jeans equation. Watkins, Evan \& An (2010) have solved the Jeans equation for the case of set of mass tracers with both distances (not projected distances) and radial velocities located in the outer halo of a massive galaxy such as the Milky Way. To accomplish this they assume that the tracers follow a power-law density distribution 
$\rho \propto r^{-\gamma}$. They further assume that a NFW halo (Navarro, Frenk \& White 1996) is an adequate representation of the outer part of the Milky Way halo.

Their result then simplifies (see Evans, An, \& Deason 2011) to

$$
M_{v i r} \approx \frac{r_{v i r}^{0.5}(0.5+|\gamma|-2 \beta)}{G N} \sum_{i=1}^{N} r_{i}^{0.5} v_{r, i}^{2} . \quad \text { (eqtn. 1) }
$$

Lacking proper motions, we cannot evaluate the velocity anisotropy $\beta$; we assume isotropic orbits $(\beta=0)$ for the outer halo RR Lyr. Attempts to measure $\beta$ in the inner halo are more successful as much larger samples of tracers can be assembled, usually SDSS BHB stars. Kafle et al. (2012), among others, suggest $\beta=+0.5$ from $\sim 25 \mathrm{kpc}$ out to the limit of their sample at $\sim 60 \mathrm{kpc}$, a value similar to that deduced by Williams \& Evans (2015), who apply a new theoretical analysis to previously published data, while Deason et al. (2011) suggest from CDM simulations that $\beta=0$.

Using the above formula with $\beta=0.0$ we find a total mass out to $110 \mathrm{kpc}$ of $\sim 7 \pm 1.5 \times 10^{11} M_{\odot}$, where the uncertainty corresponds to a range in $\beta$ from -0.5 to +0.5 . This suggests a rather low total mass for the MW, but is in reasonable agreement with several recent determinations based on studying halo stars at smaller radii (see the compiled recent measurements in Fig. 5 of Williams \& Evans 2015), although analyses including the outermost MW satellites and M31 timing arguements continue to suggest a higher total mass.

Future work should expand our sample of outer halo RR Lyr significantly. Another two years of PTF imaging (2012-2014) has recently been added to the PTF variable star database, suggesting it is time to conduct another search for candidate RR Lyr in the $\mathrm{PTF}+\mathrm{iPTF}$ database, which is a rather daunting task given the limited available human resources.

\section{References}

Brown, W. R., Geller, M. J., Kenyon, S. J., \& Diaferio, A., 2010, AJ, 139, 59

Deason, A. J., McCarthy, I. G., Font, A. S. et al. 2011, MNRAS, 415, 2607

Deason, A. J., Belokurov, V., Evans, N. W. et al. 2012b, MNRAS, 425, 2840

Deason, A. J., Belokurov, V., Koposov, S. E., \& Rockosi, C., 2014, ApJ, 787, 30

Evans, N. W., An, J., \& Deason, A. J., 2011, ApJL, 730, L26

Faber, S., Phillips, A. C., Kibrick, R. I. et al. 2003, SPIE, 4841, 1657

Gnedin, O. Y., Brown, W. R., Geller, M. J., \& Kenyon, S., 2010, ApJL, 720, L108

Kaffle, P. R., Sharma, S., Lewis, G. F., \& Bland-Hawthorn, J., 2012, ApJ, 761, A98

Kaffle, P. R., Sharma, S., Lewis, G. F. et al. 2014, ApJ, 794, A59

Law, N. M., Kulkarni, S. R., Dekany, R. G. et al. 2009, PASP, 121, 1395

Navarro, J. F., Frenk, C. S., \& White, S. M., 1996, ApJ, 462, 563

Rau, A., Kulkarni, S. R., Law, N. M. et al. 2009, PASP, 121, 1334

Schlegel, D., Finbeiner, D. P., \& Davis, M., 1998, ApJ, 500, 525

Sesar, B., 2012, AJ, 144, A114

Sesar, B., Banholzer, S. R., Cohen, J. G. et al. 2014, ApJ, 793, A135

Watkins, L. L., Evans, N. W., \& An, J. H., 2010, MNRAS, 406, 264

Williams, A. A. \& Evans, N. W., 2015, MNRAS, 454, 698

Xue, X. X., Rix, H.-W., Zhao, G. et al. 2008, ApJ, 684, 1143

Xue, X. X., Rix, H.-W., Yanny, B. et al. 2011, ApJ, 738, A79

Xue, X. X., Ma, Z., Rix, H.-W. et al. 2014, ApJ, 784, 170

Xue, X. X., Rix, H. W., Ma, Z., Morrison, H., Bovy, J., Sesar, B. \& Janesh, H., 2015, ApJ, 809, 144 